\title{
A Three-Component Model Based on Macropropagation Phenomena Approach for Complex Building Scattering
}

\author{
Mehdi Ait-Ighil, ${ }^{1}$ Fernando Perez-Fontan, ${ }^{2}$ Joel Lemorton, ${ }^{1}$ Frederic Lacoste, ${ }^{3}$ \\ Christophe Bourga, ${ }^{4}$ and Michel Bousquet ${ }^{5}$ \\ ${ }^{1}$ Onera (The French Aerospace Lab), Electromagnetism and Radar Department, Radio-Communication and
Propagation Research Unit, 2 avenue Edouard Belin, 31055 Toulouse, France
${ }^{2}$ Signal Theory and Communication Department, University of Vigo, 36310 Vigo, Spain
${ }^{3}$ Centre National d'Etudes Spatiales (CNES), 18 avenue Edouard Belin, 31400 Toulouse, France
${ }^{4}$ Thales Alenia Space, 26 avenue Jean Francois Champollion, 31100 Toulouse, France
${ }^{5}$ Institut Supérieur de l'Aéronautique et de l'Espace (ISAE), Campus Supaéro, 10 avenue Edouard Belin, 31055 Toulouse, France
}

Correspondence should be addressed to Mehdi Ait-Ighil; mehdi.ait_ighil@onera.fr

Received 28 November 2014; Accepted 7 June 2015

Academic Editor: Atsushi Mase

Copyright (C) 2015 Mehdi Ait-Ighil et al. This is an open access article distributed under the Creative Commons Attribution License, which permits unrestricted use, distribution, and reproduction in any medium, provided the original work is properly cited.

Multipaths represent a common predominant and uncontrolled component on channel impairments for all terrestrial and Land Mobile Satellite systems. Without restrictions w.r.t mobile terrestrial applications, the addressed multipath problematic in this paper is focused on Land Mobile Satellite applications where delayed signal replicas are highly impacting performances on communication systems while they induce strong positioning errors for navigation systems. The actual trend in propagation channel modelling is to improve the multipath characterisation and representation by using semideterministic and hybrid physical-statistical models into channel simulators instead of narrow-band empirical approaches. In this context, this paper presents a new simplified model, called 3CM (3-Component Model) to reproduce building scattering in an efficient way which strongly improves computation performances. This model is based on asymptotic methods, namely, PO (Physical Optics) which allows the 3CM to be frequency scalable, polarimetric, and dielectric materials oriented. Note that the proposed model and the retained approach can be integrated into more complex tools such as existing ray tracers. However, this issue is not discussed in this paper.

\section{Introduction}

Over the last twenty years, bandwidth requirements for telecommunication systems have experienced a continuous growth, for example, indoor wireless standard evolutions IEEE $802.11 \mathrm{a}, \mathrm{b}, \mathrm{g}$, and $\mathrm{n}$ or terrestrial mobile communication systems from GSM to LTE. Initially used for voice and message transmission, satellite based systems are following the same trend and are now widely used for multimedia broadcasting as in DVB-S or SDMB standards. Satellite navigation systems are another widely spread application which requires larger bandwidths with spectrum evolutions for new systems such as GPS-III (Global Positioning System) and Galileo. The common point of all above systems which are operating in $\mathrm{L}, \mathrm{S}$, and $\mathrm{C}$ bands is their sensitivity to various channel impairments induced by urban environments such as blockage, shadowing, multipath fading, delayed echoes, Doppler spreading, and depolarization. Even more, for wideband applications a particular emphasis has to be placed on MP (Multipath) characterisation and representation. For the sake of clarity, the following discussion will be focused on urban environments targeting LMS (Land Mobile Satellite) mobile applications. However, the three-macropropagationphenomena approach here presented can be reused for mobile terrestrial propagation models. Even more, the proposed model can be integrated into existing ray-tracing tools without major adjustments but this issue is out of the scope of the present paper.

Many channel simulators have been proposed to synthesise the LMS propagation channel where both accuracy and execution time are key parameters [1-4]. In such complex simulation tools, different models are usually integrated to 
compute the EM (ElectroMagnetic) interactions with all sorts of objects in the scene. In urban environments, buildings and vegetation are commonly considered as critical elements in the scene. Recent developments [5] have shown that street furniture such as lampposts, bus shelters, other vehicles, or ground reflections may also significantly contribute to such multipath environment. However, the focus of this paper is restricted to the modelling of EM interactions with the facades present into a given scene.

From the literature, three types of approaches can be found to reproduce the scattering phenomenon from complex buildings. The first category encompasses all deterministic numerical approaches from full-wave methods, for example, MoM (Method of Moments), to asymptotic methods, for example, PO (Physical Optics), GO (Geometrical Optics), or ECM (Equivalent Current Method). They present the advantage of being very versatile and already used in various simulation tools. Their main drawbacks are the computation time which may be prohibitive in complex urban environments and the need of complex environment models (geometry and material properties) to perform the EM computation. On the opposite side, the second category encompasses all statistical approaches based on the GSCM approach (Geometry-based Stochastic Channel Model) where point scatterers with empirical power profiles are spread around the mobile location $[6,7]$. Those models present the advantage of being very fast with very low computational effort. However, those models need to be tuned by measurement campaigns and can only be extrapolated to very similar environments since they are not using any EM model to predict wave interactions with the environment. In between, hybrid physical-statistical models are a tradeoff between statistical approaches and deterministic ones to keep time performances from the first and realism and versatility from the second. The retained tradeoff implies the design of a new simplified EM building scattering model which remains fast, frequency scalable, and polarisation and dielectric material oriented.

The problematic of building scattering and rough surface scattering here addressed has been previously studied in [8, 9]. Other approaches aiming at homogenizing the different dielectric material present on facade into one equivalent material have been presented in [10]. Very recent work has also been presented in [11] to discuss the polarisation issue leading to one empirical model extracted from measurement campaigns. The goal of this paper is to design a new building scattering model based on EM models with a minimum resort to empirical approaches or measurement campaigns.

The remainder of this paper is organized as follows. Section 2 presents several observations that have been made prior to designing the simplified building scattering model. Section 3 presents the fundamentals and the main principles of the 3CM (3-Component Model). Section 4 presents two narrow-band validation examples: one on a complex isolated facade and another on a simple urban canyon. Both examples have been validated considering both MoM based and asymptotic GO/PO based reference simulation tools. Section 5 presents two wide-band validation examples: one qualitative comparison between power/delay/Doppler CIRs

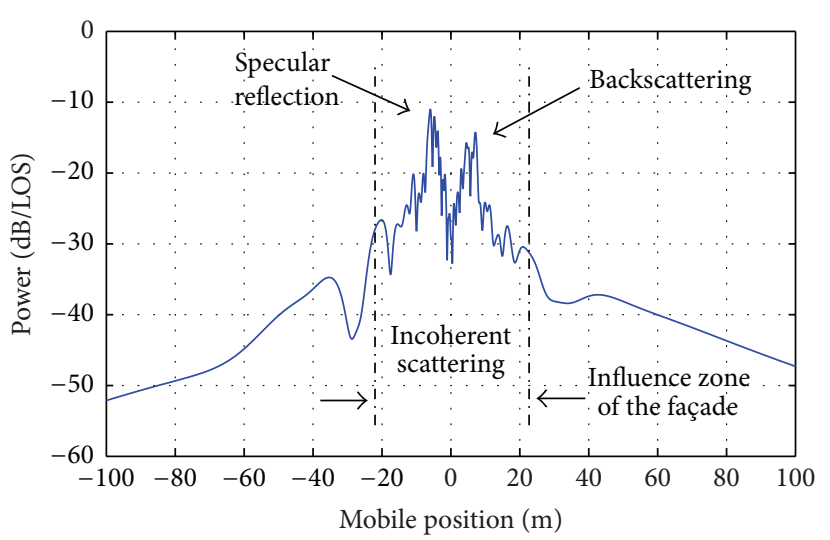

FIGURE 1: Narrow-band total scattered power received when passing next to a complex facade using ELSEM3D (MoM).

(Channel Impulse Response) and the other focused on the mean delay and delay spread. Finally, Section 6 concludes and presents some features to be added to improve the actual implemented model.

\section{Practical Observations}

2.1. Narrow-Band Observations Based on MoM Results. The first observation here presented in Figure 1 has been realised using ELSEM3D, an EM research code developed by ONERA $[12,13]$. It is based on the EFIE (Electric Field Integral Equation) solved with MoM techniques in the frequency domain. The advantages of exact methods such as MoM are that EM computation remains valid on small features and subwavelength elements. However, the size of the problem is limited to few tens of wavelengths and computation takes a very long time. As an example, the simulation on which this observation has been made has been run over 8 parallel supercomputers during $36 \mathrm{~h}$. Note that, in the following part of this paper, ELSEM3D will be used as a reference.

The scenario from which Figure 1 has been extracted is presented in Figure 2. A $4 \mathrm{~m}$ width and $6 \mathrm{~m}$ height facade (as presented in Figure 9(b)) is placed on the ground plane $Z=0$. The $X$-axis is normal to the facade while the $Z$-axis is oriented toward the sky. The bottom center of the facade is placed at $y=0$. The receiver is moving from $-100 \mathrm{~m}$ to $+100 \mathrm{~m}$ following the $Y$-axis at a constant distance $x=4 \mathrm{~m}$. In this scene, no ground effect has been taken into account due to the targeted satellite applications which basically use hemispheric antennas pointed towards the sky where echoes coming from below its horizon are attenuated by several tens of decibels. The source is placed at $20000 \mathrm{~km}, 40^{\circ}$ elevation with respect to the ground plane, and $40^{\circ}$ azimuth with respect to $X$-axis. Figure 1 represents the total power scattered by the facade when the mobile is passing nearby on a logarithm scale with respect to to the LOS level.

As visible in Figure 1, two zones can be delimited from the observation of the total scattered power, a zone where the facade has no influence (low scattered power with smooth variations) and a zone where the facade has an influence 


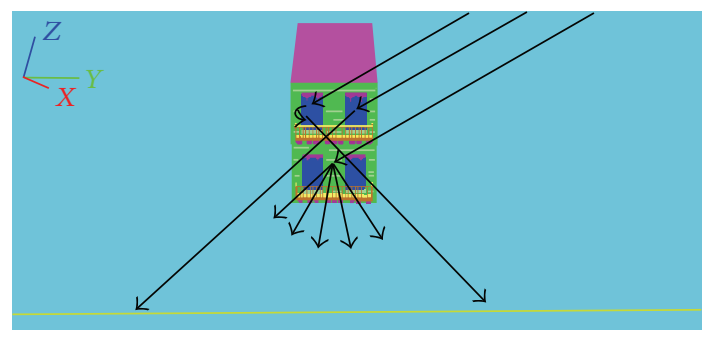

FIGURE 2: Geometrical configuration of the presented simulation.

(strong scattered power with fast variations). The influence zone of the facade can be linked to the incoherent scattering phenomenon taking place on small scatterers. This zone seems to be characterised by a power threshold around $-30 \mathrm{~dB}$ with respect to LOS with fast variations from $y=$ $-20 \mathrm{~m}$ to $y=+20 \mathrm{~m}$. Above this threshold, two peaks are clearly visible. The first one, located around $y=-5 \mathrm{~m}$, can be linked to a specular reflection phenomenon on the facade by using simple geometric relation between receiver and transmitter positions. The second peak, around $y=+5 \mathrm{~m}$, symmetrical with respect to the specular one, is slightly lower in terms of power which is linked to double bond reflection called backscattering. The physical process involved in this third propagation mechanism is detailed in Section 3.

\subsection{Wide-Band Observations Based on Asymptotic Methods} Results. The second observation here presented in Figure 3 is based on commercial asymptotic tool. SE-WorkBench software suite is codeveloped by Oktal-SE and Onera [15]. The main processing software here used is SE-Ray-EM. SE-RayEM performs all basic operations such as the SBR (Shooting and Bouncing Rays) algorithm and the use of asymptotic techniques to model EM interactions with the environment such as PO and GO for multiple reflections or ECM for edge diffraction. This main processing unit is coupled to a more specific module for propagation and channel modelling in mobile context. This wide-band module allows us to postprocess the raw EM field. Using information such as DoA (Direction of Arrival) or propagation path length of each ray, the complex CIR can be computed. To reduce the number of rays from millions to thousands, a tapped-delay line approach is used where all rays belonging to the same tap are coherently summed together. The tapped-delay line approach has been extended to three dimensions: delay, DoA azimuth, and DoA elevation, where each sampled dimension has its own resolution. Note that, in the following, SE-WorkBench will be used as a second reference.

The scenario from which Figure 3 has been extracted is similar to the one presented previously: a mobile receiver is moving into a simple urban canyon made of several facades and buildings. Only one side of the street is filled by buildings. The other one is building-free. The transmitter is placed at $20000 \mathrm{~km}, 40^{\circ}$ elevation, and $40^{\circ}$ azimuth. The receiver is moving at a constant speed of $50 \mathrm{~km} / \mathrm{h}$ parallel to the building row. In those conditions, perfect LOS is achieved all along receiver's trajectory. Figure 3 represents one example of

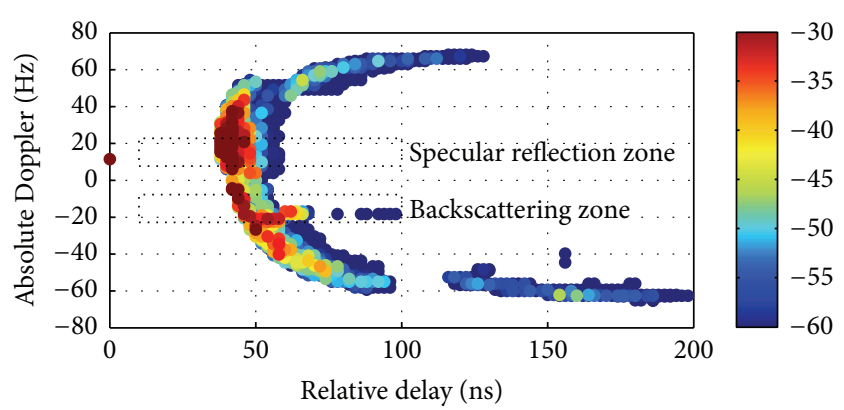

FIGURE 3: Wide-band delay/Doppler channel IR when passing in a simple urban canyon using asymptotic GO/PO tools.

instantaneous CIR. The abscissa axis represents the relative delay of the echoes with respect to the LOS delay. The ordinate axis represents the absolute Doppler of each echo assuming a fixed transmitting point. The colour axis represents the power of each echo with respect to the LOS power.

From Figure 3, we can first isolate the LOS contribution with null relative delay and positive Doppler. From the overall MP spread, two powerful zones can be isolated. The first one has a positive Doppler of around $15 \mathrm{~Hz}$. This zone contains the strongest echoes. The positive Doppler permits linking this zone to a specular phenomenon coming from the front of the receiver. The second zone is less powerful with negative Doppler. Still, this zone contains significant echoes. The negative Doppler and the symmetry with respect to the specular zone indicate some kind of backward reflection coming from the rear of the receiver, previously called backscattering phenomenon. The influence zone of the street canyon can also be highlighted from $-40 \mathrm{~Hz}$ to $+40 \mathrm{~Hz}$. Note that the shape of delay/Doppler spread is site dependent and tightly linked to the canyon geometry. To conclude this observation, a parallel can easily be made with the first observation made in Section 2.1 for the specular, backscattering, and incoherent scattering zones.

2.3. DoA Observation Based on Measurement Campaign. The last observation here presented in Figure 4 is based on a measurement campaign realised by DLR (German Aerospace Center) in 2002. The measurements aimed at providing real data to build an enhanced physical-statistical LMS channel model designed for satellite navigation purposes [7] and later referenced by ITU [2]. Those measurements have been performed by using a Zeppelin for transporting the transmitter and a moving car carrying the receiver. Several environment types have been investigated such as rural, suburban, or urban. From those data, various channel parameters were extracted such as the PDF (Probability Density Function) of the scatterer position, scatterer lifespan, reflected mean power of scatterers, or the number of significant echoes.

Figure 4 represents a PDF of the scatterer positions for urban environment. In the presented case, the LOS is coming with $25^{\circ}$ azimuth and $30^{\circ}$ elevation and the mobile is moving along the $X$-axis. Three zones have been highlighted in Figure 4. They represent specular, backscattering, and incoherent scattering propagation phenomena, already observed 


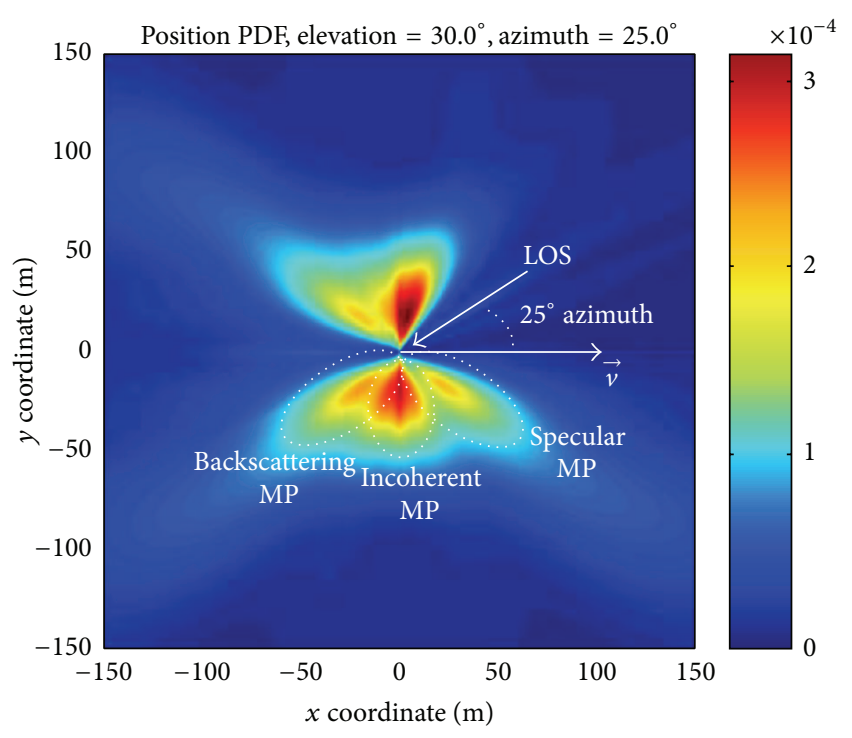

Figure 4: Position probability density function of scatterers extracted from measurements DLR 2002 [14].

on previous sections. From an angular point of view, the specular contribution is symmetrical to the LOS with respect to receiver motion $\vec{v}$, the backscattering contribution comes from the opposite direction with respect to the LOS, and the incoherent contribution makes the transition between both.

In the data analysis made in [14], the authors pointed out some double reflection effects taking place mainly in urban environments which lead to backward propagation schemes. Those observations confirm our preliminary interpretations made in Sections 2.1 and 2.2.

\section{Proposed Modelling Approach}

3.1. Description of the Three-Component Model (3CM). From the previous observations made in Section 2, it has been shown that the building scattering phenomenon taking place around complex facades can be broken down into three different propagation mechanisms. Those three propagation mechanisms represent each of the three components on which the 3CM lies. They are illustrated in Figure 5 and detailed below.

(i) The specular component reproduces the forward reflection phenomenon taking place on wide and smooth surfaces such as windows and flat walls. This component is the most powerful. Its implementation is based on a simplified version of the PO algorithm detailed in Section 3.2.

(ii) The backscattering component comes from a double bounce reflection, or backward reflection, due to dihedral effects taking place near protruding and receding elements of the facade, that is, receding windows or protruding balconies. The backscattering component is less powerful than the specular reflection but is responsible for significant contributions coming from the opposite side of the LOS

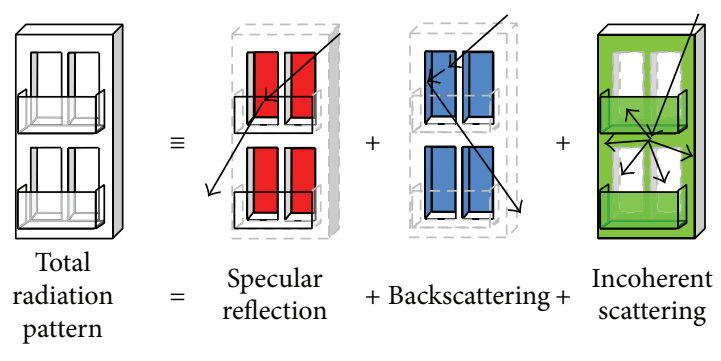

Figure 5: Decomposition principle of the 3CM approach.

contribution. From existent models, this component was usually integrated into the incoherent scattering phenomenon although the EM mechanism is coherent and very different. The implementation of the backscattering component is based on the equivalent plate concept detailed in Section 3.3.

(iii) The incoherent scattering component reproduces the scattering phenomenon due to small details present on facades and surface roughness. It also characterises the influence zone of the facade. Since small features are not supposed to be modelled using asymptotic methods such as PO, the implementation of this component is based on analytical models extracted from [16] and detailed in Section 3.4.

In this hybrid physical-statistical approach, both coherent components, namely, specular reflection and backscattering, are macroscale scattering phenomena which are mainly linked to the architecture of the facade and modelled using EM deterministic methods, namely, PO. On the contrary, the incoherent scattering is a microscale scattering phenomenon which is linked to the presence of small scatterers and to the surface roughness and modelled using empirical models. The main asset of such modelling is to jointly compute macroand microscale scattering with lower computation times. In fact, the total scattering pattern of any complex facade can be broken down into those three components, with each one being easy to model. One other advantage of the $3 \mathrm{CM}$ is its physical EM base which gives it more realism and versatility. Contrary to empirical models used alone, the 3CM remains linked to the building geometry. Even more, since it is based on the PO algorithm, the 3CM can also deal with dielectric materials, can handle circular polarisation, and is frequency scalable at least from 1 to $5 \mathrm{GHz}$ in its current implementation. Note that, for higher frequencies, the $3 \mathrm{CM}$ is not restricted in its approach but would require more detailed environment as inputs according to [17] which have not been investigated yet.

In the next sections, the EM basis of each three components is detailed.

3.2. The Specular Component. The specular component is based on a simplified PO algorithm inspired from [18] and presented next. The first step of the PO algorithm is to compute tangential $\vec{E}^{t}$ and $\vec{H}^{t}$ fields. $E$ represents the electric field, $H$ represents the magnetic field, superscript $i$ is for incident field, $r$ is for geometrically reflected field, and $t$ is for 
tangential field over the surface $S$. Not detailed here, incident fields are polarisation dependent and reflected fields depend on both Fresnel reflection coefficients, $R_{\|}$and $R_{\perp}$, applied to the incident polarised fields:

$$
\begin{aligned}
\vec{E}^{t} & =\vec{E}^{i}+\vec{E}^{r}, \\
\vec{H}^{t} & =\vec{H}^{i}+\vec{H}^{r} .
\end{aligned}
$$

From the tangential EM fields, surface currents are calculated. Note that EM fields and surface currents are expressed in the local xyn reference base of the facet. $J_{S}$ represents the electric surface current while $M_{S}$ represents the magnetic surface current. $\hat{n}$ represents the unit normal vector to the facet:

$$
\begin{aligned}
\vec{J}_{s} & =\widehat{n} \wedge \vec{H}^{t}, \\
\vec{M}_{s} & =-\widehat{n} \wedge \vec{E}^{t} .
\end{aligned}
$$

EM surface currents are then reradiated using (3) where $\beta$ is the wave number, $\widehat{k}^{s}$ is the unit scattering direction, and $\widehat{k}^{i}$ is the unit incident direction both $\widehat{k}^{i}$ and $\widehat{k}^{s}$ unit vectors expressed in the local facet base:

$$
\begin{aligned}
\vec{N} & =\iint_{S} \vec{J}_{s} e^{+\jmath \beta\left(\widehat{k}^{s}-\widehat{k}^{i}\right) \wedge \widehat{n}} d s^{\prime}, \\
\vec{L} & =\iint_{S} \vec{M}_{s} e^{+\jmath \beta\left(\widehat{k}^{s}-\widehat{k}^{i}\right) \wedge \widehat{n}} d s^{\prime} .
\end{aligned}
$$

The simplified denomination here used for the PO algorithm comes from the fact that, instead of solving the integral equations presented in (3), a square plate of width $w$ is assumed and the integral term becomes a sinc function as shown in

$$
\int_{-w / 2}^{+w / 2} e^{\jmath \alpha z} d z=w\left[\frac{\sin ((\alpha / 2) w)}{(\alpha / 2) w}\right]=w \operatorname{sinc}\left(\frac{\alpha}{2} w\right) .
$$

Using projection relations, $\vec{N}$ and $\vec{L}$ lead to (5) to (8) using notations described in (9) and (10), $\lambda$ represents the wavelength, and $\widehat{\theta}$ and $\widehat{\phi}$ represent unit polarisation vectors:

$$
\begin{aligned}
N_{\theta} & =\left(\widehat{\theta} \cdot \vec{J}_{s}\right) \prod_{x y} \operatorname{sinc}(\vec{\xi}) w^{2}, \\
N_{\phi} & =\left(\widehat{\phi} \cdot \vec{J}_{s}\right) \prod_{x y} \operatorname{sinc}(\vec{\xi}) w^{2}, \\
L_{\theta} & =\left(\widehat{\theta} \cdot \vec{M}_{s}\right) \prod_{x y} \operatorname{sinc}(\vec{\xi}) w^{2}, \\
L_{\phi} & =\left(\widehat{\phi} \cdot \vec{M}_{s}\right) \prod_{x y} \operatorname{sinc}(\vec{\xi}) w^{2}, \\
\prod_{x y} \operatorname{sinc}(\vec{\xi}) & \equiv \operatorname{sinc}\left(\vec{\xi} \cdot \widehat{e}_{x}\right) \operatorname{sinc}\left(\vec{\xi} \cdot \widehat{e}_{y}\right), \\
\vec{\xi} & =\frac{\pi w}{\lambda}\left(\widehat{k}^{s}-\widehat{k}^{i}\right) \wedge \widehat{n} .
\end{aligned}
$$

Finally, the $E$ and $H$ fields along both $\widehat{\theta}$ and $\widehat{\phi}$ polarisation vectors are calculated using (5) to (8):

$$
\begin{aligned}
& E_{\theta}=-\frac{\jmath \beta e^{-\jmath \beta r}}{4 \pi r}\left(L_{\phi}+\eta N_{\theta}\right), \\
& E_{\phi}=+\frac{\jmath \beta e^{-\jmath \beta r}}{4 \pi r}\left(L_{\theta}-\eta N_{\phi}\right), \\
& H_{\theta}=+\frac{\jmath \beta e^{-\jmath \beta r}}{4 \pi r}\left(N_{\phi}-\frac{L_{\theta}}{\eta}\right), \\
& H_{\phi}=-\frac{\jmath \beta e^{-\jmath \beta r}}{4 \pi r}\left(N_{\theta}+\frac{L_{\phi}}{\eta}\right) .
\end{aligned}
$$

This implementation of the specular component has been validated using SE-WorkBench reference with different constraints such as bistatic reflection configurations and polarimetric simulations. Two examples are presented in Figure 6.

Figure 6(a) represents the RCS (Radar Cross Section) of a dielectric plate. The source is placed at $20000 \mathrm{~km}$ from the center of the plate with normal incidence and $\theta$ polarisation with a center frequency of $1.5 \mathrm{GHz}$. The plate is a $2 \mathrm{~m}$ by $2 \mathrm{~m}$ dielectric square with the following dielectric constant: $\epsilon_{r}=$ 3.7, $\mu_{r}=1$, and $\sigma=0$ representative of brick at this frequency. The plate is subdivided into smaller square facets of $0.5 \mathrm{~m}$ width. The RCS is measured at $100 \mathrm{~m}$ from the plate center in the horizontal plane. As visible, the simplified PO algorithm is in good accordance with the reference made using SEWorkBench. Both simulations are also in accordance with theoretical results which predict an RCS of $27 \mathrm{dBm}^{2}$ in the normal direction using (12), where $S$ represents the surface of the plate:

$$
\begin{aligned}
\operatorname{RCS} & =\frac{\rho^{2}\left(4 \pi S^{2}\right)}{\left(\lambda^{2}\right)}, \\
\rho & =\frac{\left(\sqrt{\epsilon_{r}}-1\right)}{\left(\sqrt{\epsilon_{r}}+1\right)} .
\end{aligned}
$$

Figure 6(b) represents the total power reflected by a PEC (Perfect Electric Conductor) plate in the near field. The source is placed at $20000 \mathrm{~km}$ from the center of the plate with $50^{\circ}$ elevation, $15^{\circ}$ azimuth, and $\theta$ polarisation. The plate is a $2 \mathrm{~m}$ by $2 \mathrm{~m}$ PEC square. The plate is subdivided into smaller square facets of $0.5 \mathrm{~m}$ width. The far field limit for the whole plate is around $80 \mathrm{~m}$ while the far field of the smaller facets is $5 \mathrm{~m}$. The total reflected field is measured at $10 \mathrm{~m}$ with constant $\theta=140^{\circ}$ and $\phi$ varying from $-90^{\circ}$ to $+90^{\circ}$. Note that the PO formulation used has to fulfil far field conditions, meaning that each receiving point has to be in far field with respect to the subdivided element of side $0.5 \mathrm{~m}$ in this case. Here also, a good agreement is achieved between SE-WorkBench reference and the $3 \mathrm{CM}$.

3.3. The Backscattering Component. The first attempt to model the backscattering component was based on a hybrid 


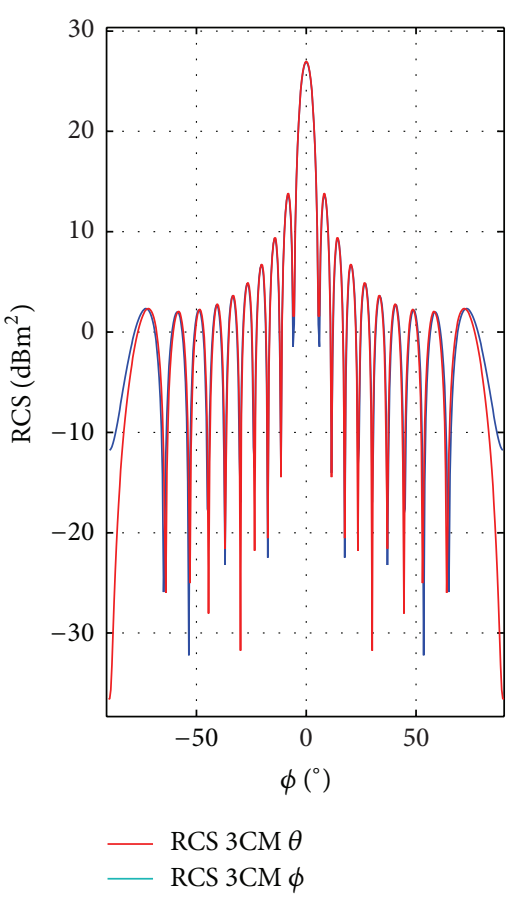

(a) Dielectric plate RCS

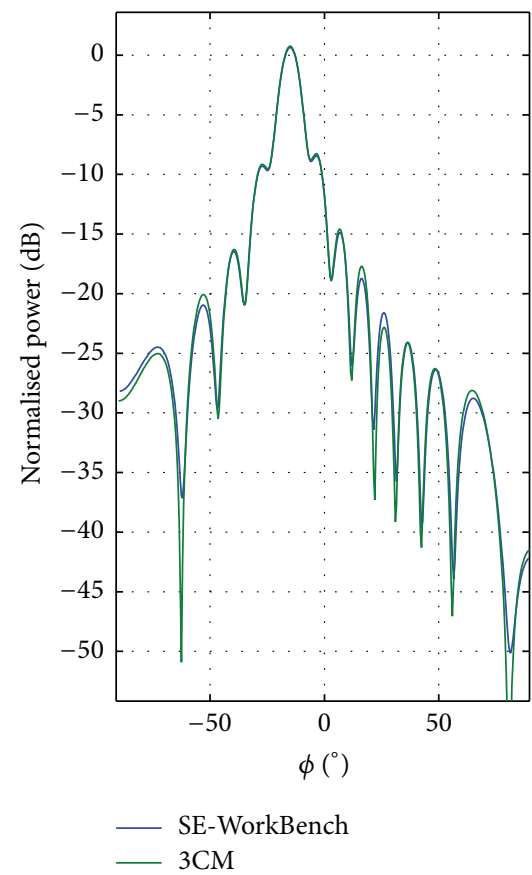

(b) Near field reflection on PEC plate

FIGURE 6: Validation examples of the implemented simplified PO algorithm for the specular component.

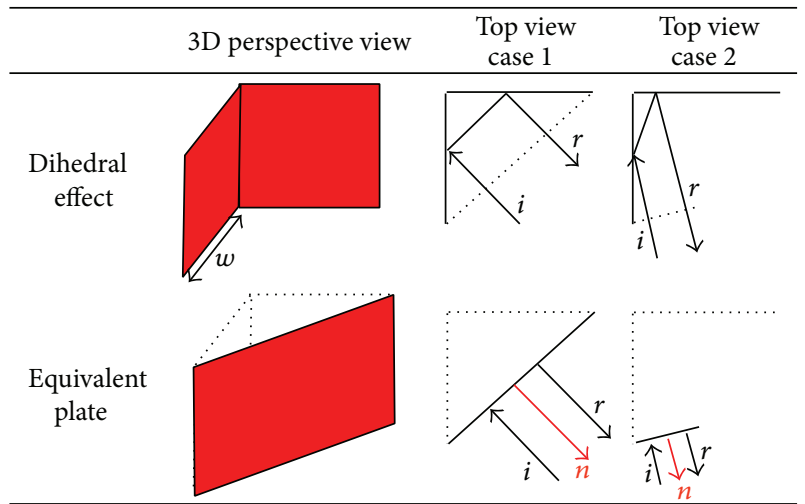

FIGURE 7: Principle of the equivalent plate model used to reproduce the backscattering component.

GO-PO algorithm which rigorously models the double reflection phenomenon as done by SE-WorkBench. This solution turns out to be very expensive in terms of computation time and has been abandoned. The present version of the backscattering component lies on an equivalent plate model. The principle is to replace any dihedral by an equivalent plate in terms of size and orientation on which the simplified PO algorithm previously presented can easily be applied. Figure 7 illustrates the concept.

In fact, one of the properties of the dihedral is to reflect the energy where it comes in the horizontal plane, with the propagation in the horizontal plane remaining unchanged. A plate oriented toward this direction would then reflect this energy in this exact precise direction. The orientation of the plate corresponds to a rotation in the horizontal plane which satisfies

$$
\widehat{n}=-\frac{1}{\sqrt{\left(k_{x}^{i}\right)^{2}+\left(k_{y}^{i}\right)^{2}}}\left(\begin{array}{c}
k_{x}^{i} \\
k_{y}^{i} \\
0
\end{array}\right),
$$

where $k_{x}^{i}$ and $k_{y}^{i}$ represent the $x$ and $y$ components of $k^{i}$ incident vector in the global reference base of the scene.

About the size of the equivalent plate, because of the azimuth angle of the incoming rays, some parts of the dihedral are not physically illuminated which reduces the visible section of the dihedral. To reproduce this phenomenon, a coefficient is applied to the width of the facet as presented in

$$
w^{\prime}=2 w \min \left(\cos \phi^{i}, \sin \phi^{i}\right) \cos ^{3} \theta^{i},
$$

where $w^{\prime}$ represents the equivalent width of the plate, $w$ the original width of the plate, $\phi^{i}$ the incident azimuth angle, and $\theta^{i}$ the incident elevation angle.

To replace the double interaction phenomenon by a simple interaction model using the simplified PO algorithm, the first reflection has to be taken into account. To do so and before computing the reflection on the equivalent plate by using the $\mathrm{PO}$ algorithm, a prereflection is computed by applying a polarisation rotation assuming a PEC material and a constant attenuation to reproduce the dielectric material impact on the first reflection.

Finally, the radiation pattern of the equivalent plate is empirically corrected to reproduce the side lobes around $-30^{\circ}$ 


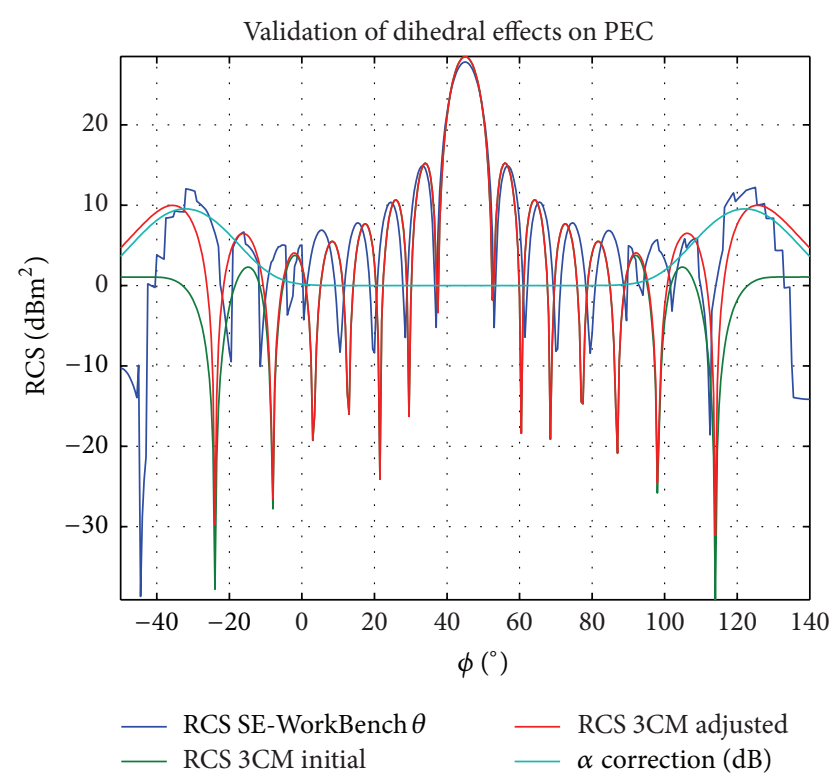

FIGURE 8: Validation example of the backscattering component implementation.

and $+125^{\circ}$ as visible in Figure 8 due to the edge diffractions of the dihedral which are not taken into account by the equivalent plate model. The empirical correction function $\alpha$ is presented in (16). Note that, out of $\left[-50^{\circ} ;+140^{\circ}\right]$ interval, the correction function equals zeros avoiding the backscattering component to radiate in a nonphysical direction:

$$
\alpha=1+\sin \left[\left(\phi^{s}-\frac{\pi}{4}\right) \kappa\right]^{20},
$$

where $\phi^{s}$ is the scattering azimuth angle and $\kappa=105 / 90$ is an empirical broadening coefficient.

Figure 8 presents one validation example for a PEC dihedral composed of two orthogonal plates of $1 \mathrm{~m}$ by $1 \mathrm{~m}$ each oriented according to $\hat{x}$ and $\hat{y}$ directions similar to the one presented in Figure 7. The source is placed at $20000 \mathrm{~km}$ in the horizontal plane with $45^{\circ}$ azimuth and $\theta$ polarised at a center frequency of $1.5 \mathrm{GHz}$. The RCS is measured in the horizontal plane from $-50^{\circ}$ to $+140^{\circ}$.

Even if empirically designed, the backscattering component is still suitable for dielectric materials including polarisation effects. The backscattering implementation has been also validated for various incidence angles with a reasonable agreement with the reference while preserving time performances.

3.4. The Incoherent Scattering Component. The incoherent scattering component is based on analytical formulas extracted from [16, chapter 9]. Originally designed for sea surface modelling, two rough surface models have been implemented. The first one corresponds to very rough surfaces with an exponential distribution. This model has a twoparameter dependence: $h$, the RMS roughness height, and $l$, the correlation length of the surface roughness. This model is based on normalised RCS, represented by $\gamma_{p q}^{I}$ parameter in (18). The total radiated field is then calculated using (17) where $S$ is the total surface of the facet, $Z_{0}$ is the vacuum impedance, $R_{1}$ and $R_{2}$ are, respectively, the distance between the transmitter and the facet and the distance between the facet and the receiver, $\theta_{i}$ is the incidence angle taken with respect to the normal of the facet in the plane $(-X Z), \phi_{s}$ is the scattering azimuth angle taken in the $(X Y)$ plane positive from $X$-axis to $Y$-axis, $\theta_{s}$ is the scattering incidence angle taken with respect to the normal of the facet, and $\widehat{\theta}$ and $\widehat{\phi}$ are the polarisation vectors:

$$
\begin{aligned}
\vec{E} & =\frac{S \sqrt{2 Z_{0}}}{4 \pi R_{1} R_{2}}\left[\begin{array}{c}
-\jmath \gamma_{p q} e^{-\jmath k_{0}\left(R_{1}+R_{2}\right)} \hat{\theta} \\
\jmath \gamma_{p q} e^{-\jmath k_{0}\left(R_{1}+R_{2}\right)} \hat{\phi}
\end{array}\right], \\
\gamma_{p q}^{I} & =\left|\beta_{p q}\right|^{2} J .
\end{aligned}
$$

$J$ function presented in (19) assumes an exponential distribution [16]:

$$
J=\frac{12}{s^{2} \xi_{z}^{2}} \exp \left[-\frac{\sqrt{6}}{s^{2}} \sqrt{\frac{\xi_{x}^{2}+\xi_{y}^{2}}{\xi_{z}^{2}}}\right]
$$

with

$$
\begin{aligned}
& s^{2}=\frac{4 h^{2}}{l^{2}}, \\
& \xi_{x}=\sin \theta_{i}-\sin \theta_{s} \cos \phi_{s}, \\
& \xi_{y}=\sin \theta_{s} \sin \phi_{s}, \\
& \xi_{z}=-\cos \theta_{i}-\cos \theta_{s} .
\end{aligned}
$$

$B$ coefficients represent the polarisation dependence of the model and are listed below:

$$
\begin{aligned}
\beta_{v v} & =\frac{a_{2} a_{3} R_{\|}(\iota)+\sin \theta_{i} \sin \theta_{s} \sin ^{2} \phi_{s} R_{\perp}(\iota)}{a_{1} a_{4}}, \\
\beta_{h v} & =\sin \phi_{s} \frac{-\sin \theta_{i} a_{3} R_{\|}(\iota)+\sin \theta_{s} a_{2} R_{\perp}(\iota)}{a_{1} a_{4}}, \\
\beta_{v h} & =\sin \phi_{s} \frac{\sin \theta_{s} a_{2} R_{\|}(\iota)-\sin \theta_{i} a_{3} R_{\perp}(\iota)}{a_{1} a_{4}}, \\
\beta_{h h} & =\frac{-\sin \theta_{i} \sin \theta_{s} \sin ^{2} \phi_{s} R_{\|}(\iota)-a_{2} a_{3} R_{\perp}(\iota)}{a_{1} a_{4}}, \\
\cos \iota & =\frac{1}{\sqrt{2}} \sqrt{1-\sin \theta_{i} \sin \theta_{s} \cos \phi_{s}+\cos \theta_{i} \cos \theta_{s},} \\
a_{1} & =1+\sin \theta_{i} \sin \theta_{s} \cos \phi_{s}-\cos \theta_{i} \cos \theta_{s}, \\
a_{2} & =\cos \theta_{i} \sin \theta_{s}+\sin \theta_{i} \cos \theta_{s} \cos \phi_{s}, \\
a_{3} & =\sin \theta_{i} \cos \theta_{s}+\cos \theta_{i} \sin \theta_{s} \cos \phi_{s}, \\
a_{4} & =\cos \theta_{i}+\cos \theta_{s} .
\end{aligned}
$$

The second incoherent scattering model is based on the PO algorithm and targets slightly rough surfaces with 


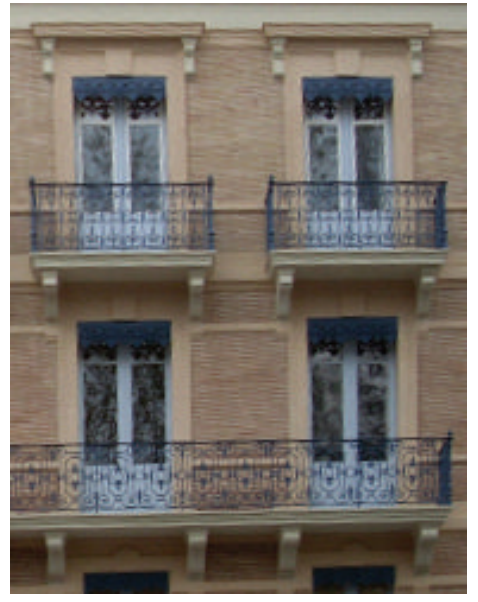

(a) Real facade

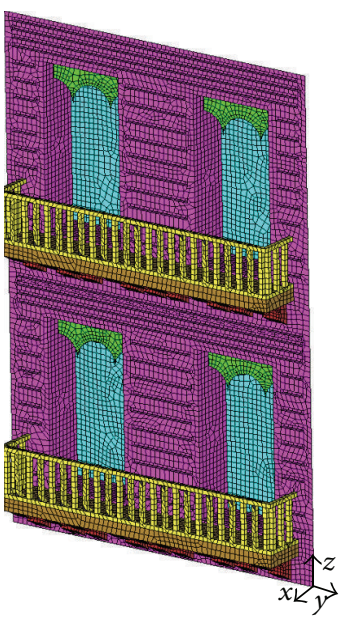

(b) 3D CAD model

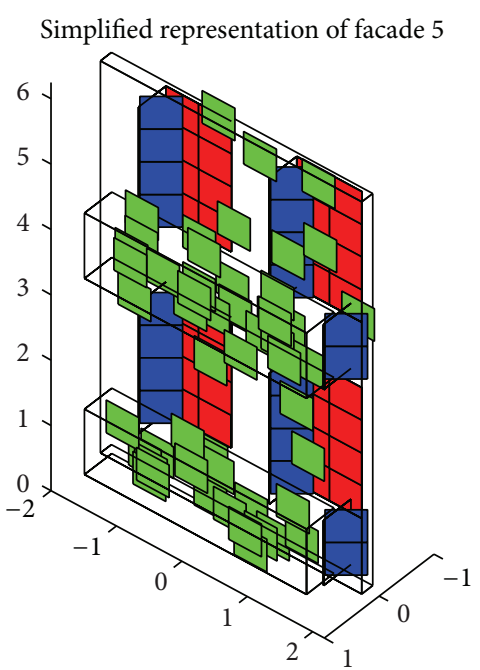

(c) Simplified 3CM

Figure 9: Three different representations of one complex city center facade.

a uniform distribution. The concept of this second model is based on the approach presented in [16], where the PO algorithm is applied to randomly generated scatterers reproducing a given roughness profile. Note that the previously mentioned scattering model is a generalised analytical model reproducing this experiment with an exponential distribution of the scatterers. This second model is called "slightly rough specular."

\section{Validation of Total Scattered Power}

4.1. Validation on Isolated Facade. This first validation subsection is relative to the total power scattered by a section of an isolated complex facade. As presented in Figure 9, several representations of the same facade are possible. Figure 9(a) is a photography of a real facade as it can be found in the city center of Toulouse, France. Figure 9(b) is an interpreted 3D CAD (Computer Aided Design) version of the same facade. This is the representation that has been used in SE-WorkBench and ELSEM3D when performing the reference simulations presented in the following. This facade is $4 \mathrm{~m}$ in width by $6 \mathrm{~m}$ in height and composed of triangles with 715000 edges. Due to restrictions on computation time when using ELSEM3D (MoM based) on such a huge object with respect to $\lambda$, a PEC material was assumed. Note that all simulations presented in the following were performed without ground and using PEC material although the 3CM perfectly handles dielectric materials. Figure 9(c) represents the same facade translated into the $3 \mathrm{CM}$ formalism. This facade is composed of twelve architectural elements, with each one being discretised into square facets of $0.5 \mathrm{~m}$ width. The size of the facets has been chosen to be maximum, to reduce computation times, but must satisfy the far field assumption mentioned above. A $0.5 \mathrm{~m}$ side is a good tradeoff for $1.5 \mathrm{GHz}$ systems and large enough and is satisfying the far field condition at $5 \mathrm{~m}$. The specular reflection model is applied to the red facets. The backscattering component is applied to the blue facets. The incoherent scattering model is applied to the green facets. As visible, the $3 \mathrm{CM}$ simplified version of this building has four windows on which the specular component is applied. It also has six backscatterers, four for receding window corners and two for protruding balconies. This facade also has two guardrails modelled using the very rough surface model with exponential roughness distribution on each facet with parameters $h=0.2$ and $l=0.5$. Those facets are randomly thrown in a volume of depth equal to $\lambda$. Finally the wall is modelled using the slightly rough specular model. This surface has not been modelled using pure specular facets since the real wall is not perfectly smooth. Using the slightly rough specular model permits breaking the phase coherence to reduce the specular phenomenon and still reproduce the incoherent scattering phenomenon.

This city center facade has been used to perform the next validation example, presented in Figure 10. The simulation scenario is similar to the one presented in Section 2.1. The facade is placed above the ground plane at position $y=0$. The transmitter is placed at $20000 \mathrm{~km}$ from the facade center. The transmitting frequency is set to $1.5 \mathrm{GHz}$. Four different incidence angles were used. A mobile receiver is moving following a linear trajectory passing in front of the facade at $x=10 \mathrm{~m}$. Figure 10 presents the total power scattered by the facade, with respect to the LOS incident power, as a function of the satellite incidence. Three results are displayed on each plot: ELSEM3D which is based on the MoM method, SEWorkBench which is based on asymptotic methods, and the 3CM.

The critical validation zone is placed between $0 \mathrm{~dB}$ and $-20 \mathrm{~dB}$ with respect to the LOS level since MP in this range has a significant impact on LMS systems. The range from $-20 \mathrm{~dB}$ to $-40 \mathrm{~dB}$ is also important in case of LOS blockage as it can frequently occur in urban environments. However, this 

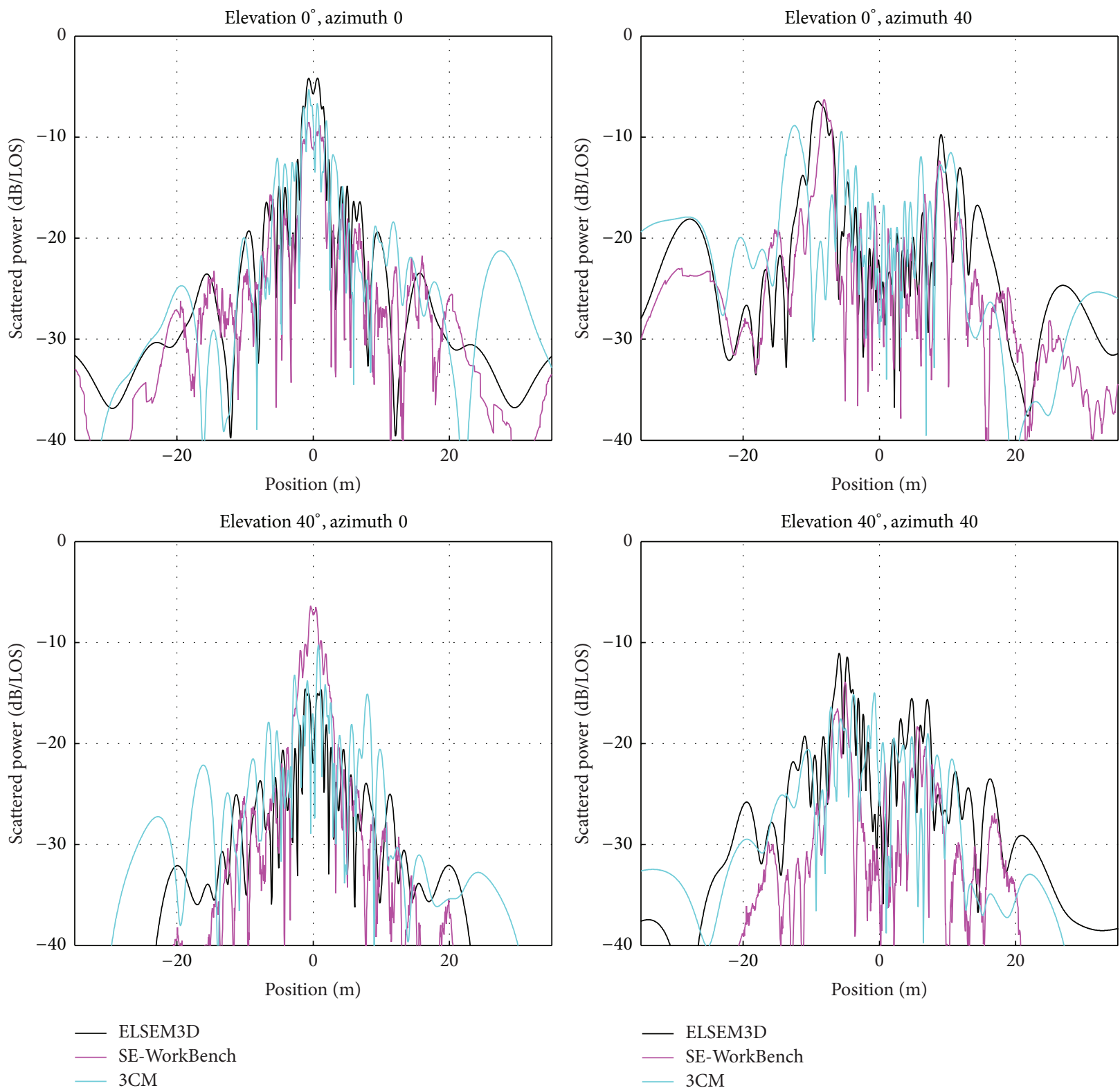

FIGURE 10: Total narrow-band power scattered by a complex facade for various incidence angles.

TABLE 1: Mean power on the interval $[0 \mathrm{~dB}-20 \mathrm{~dB}]$ (left) and on the interval $[-20 \mathrm{~dB}-40 \mathrm{~dB}]$ (right).

\begin{tabular}{lccc}
\hline & ELSEM3D & SE-WB & 3CM \\
\hline Conf. $\left(0^{\circ}, 0^{\circ}\right)$ & $-14.12,-30.1$ & $-14.60,-32.0$ & $-14.77,-31.8$ \\
Conf. $\left(0^{\circ}, 40^{\circ}\right)$ & $-15.36,-26.6$ & $-15.77,-27.9$ & $-15.92,-26.6$ \\
Conf. $\left(40^{\circ}, 0^{\circ}\right)$ & $-16.52,-36.3$ & $-11.01,-41.4$ & $-16.65,-32.9$ \\
Conf. $\left(40^{\circ}, 40^{\circ}\right)$ & $-16.74,-31.3$ & $-17.34,-37.5$ & $-17.61,-34.3$ \\
\hline
\end{tabular}

second range is less critical in terms of validation. To better quantify the differences between all three methods, mean and standard deviations have been computed over both validation intervals and presented in Tables 1 and 2, respectively.
TABLE 2: Std. of power on the interval $[0 \mathrm{~dB}-20 \mathrm{~dB}]$ (left) and on the interval $[-20 \mathrm{~dB}-40 \mathrm{~dB}]$ (right).

\begin{tabular}{lccc}
\hline & ELSEM3D & SE-WB & $3 \mathrm{CM}$ \\
\hline Conf. $\left(0^{\circ}, 0^{\circ}\right)$ & $5.3, \mathbf{4 . 6}$ & $4.4, \mathbf{7 . 6}$ & $4.3, \mathbf{7 . 1}$ \\
Conf. $\left(0^{\circ}, 40^{\circ}\right)$ & $4.0, \mathbf{4 . 0}$ & $3.8, \mathbf{5 . 0}$ & $3.4, \mathbf{5 . 3}$ \\
Conf. $\left(40^{\circ}, 0^{\circ}\right)$ & $1.7, \mathbf{8 . 9}$ & $4.4, \mathbf{1 0 . 6}$ & $3.2, \mathbf{6 . 7}$ \\
Conf. $\left(40^{\circ}, 40^{\circ}\right)$ & $2.5, \mathbf{6 . 5}$ & $1.9, \mathbf{8 . 4}$ & $1.8, \mathbf{9 . 0}$ \\
\hline
\end{tabular}

From a qualitative analysis, all three models present a very good agreement in terms of scattering shape. For all nonzero azimuth configurations (Figure 10, right), the scattering pattern reveals two significant peaks, one for the specular reflection and the other one for the backscattering. 


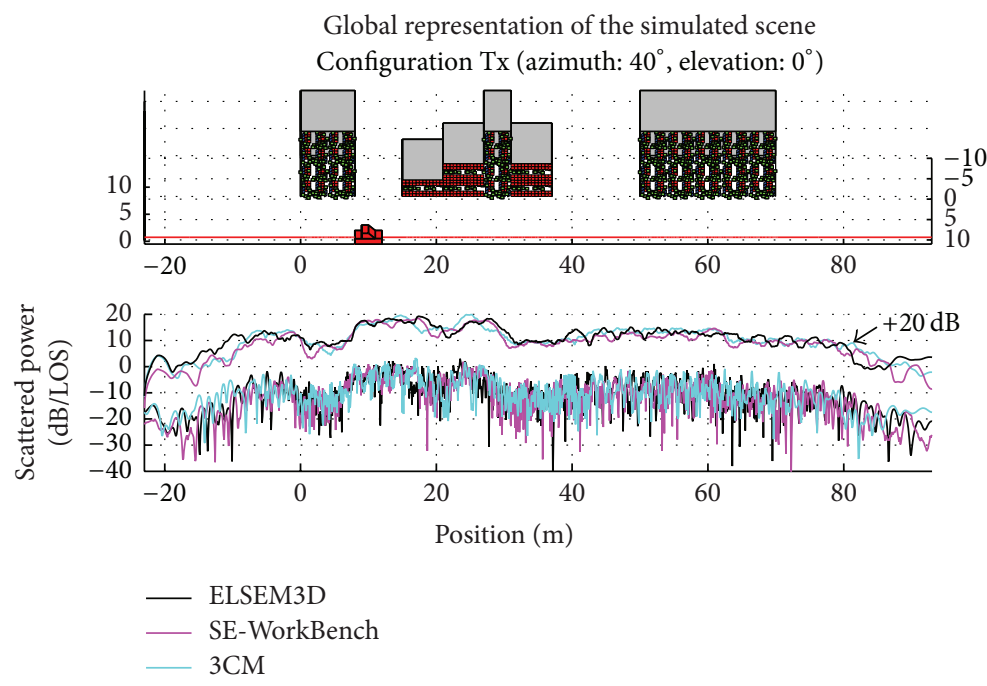

Figure 11: Total MP power scattered by a simple urban canyon.

Note that the backscattering peak is less pronounced for higher elevations. Not presented here but simulated, the 3CM model outperforms asymptotic tools for higher elevation and higher azimuth cases. This observation can be explained by the fact that the asymptotic tool tends to underestimate the incoherent scattering phenomenon which is well reproduced by the surface roughness models used in the 3CM. In terms of power, all three models are also in very good agreement and differences of the mean power in the interval $[0 \mathrm{~dB}-20 \mathrm{~dB}]$ are lower than $1 \mathrm{~dB}$, so is the standard deviation of the power. Differences are more pronounced on the interval $[-20 \mathrm{~dB}$ $-40 \mathrm{~dB}$ ] but remaining in a reasonable interval.

4.2. Validation on a Simple Canyon Configuration. The second validation example, performed in this section, consists in associating several facades with a wider scenario to represent a simple row of buildings. The simplified representation of this urban canyon used for the $3 \mathrm{CM}$ simulation is presented on top of Figure 11. Equivalent environments have been built for ELSEM3D and SE-WorkBench using 3D CAD facades such as the one presented in Figure 9(b).

The simulation scenario is as follows. A street canyon is composed of several facades. The right side of the street is building-free. The mobile receiver is following a linear trajectory at $10 \mathrm{~m}$ from the building row at a constant speed equal to $30 \mathrm{~km} / \mathrm{h}$. Bottom of Figure 11 represents the original total MP power scattered by the facades with respect to the LOS contribution in a logarithm scale. Note that the LOS contribution has not been included in this representation to better observe the MP power. With $+20 \mathrm{~dB}$ offset, a filtered representation of the same total MP power is plotted to better represent slow variations and MP macrostates of the channel. The filtering process has been realised using a sliding window of $2 \mathrm{~m}$ width which represents about $20 \lambda$.

Considering the original total MP power, all three timeseries present a reasonable agreement in terms of dynamics. However, the influence of the environment and the MP macrostate of the channel are better seen on the filtered version of the channel. From $-10 \mathrm{~m}$ to $+0 \mathrm{~m}$ and from $+40 \mathrm{~m}$ to $+60 \mathrm{~m}$ the specular reflection, respectively, on the first and third buildings is visible. From $+60 \mathrm{~m}$ to $+80 \mathrm{~m}$, a relatively high power region can be noticed. This region can be attributed to the backscattering phenomenon of the third building before decreasing after $+80 \mathrm{~m}$. A particularly powerful contribution can be noticed from $+15 \mathrm{~m}$ to $+30 \mathrm{~m}$ due to a specular reflection taking place on the second building. As visible on Figure 11, the second building is made of a different facade profile, detailed in [17], which mainly consists of specular elements. It results in higher scattered power in this zone compared to what it is near the first and third buildings. As visible, all three simulations present a reasonably good agreement in terms of mean power scattered by the canyon.

From the original complex time series, a spectrogram can be produced using the available phase information of the channel. Note that the LOS signal is still absent from those simulations and only the scattered power is considered. Figure 12 presents an interesting comparison point between a conventional ray-tracing tool such as SE-WorkBench and 3CM on a spectrogram. The method here used is based on the short-time Fourier transform. The power colour scale is given in absolute $\mathrm{dB}$ values assuming EIRP $=1 \mathrm{~W}$ at the transmitter side. From a first observation, a good agreement is noticeable. On both figures, the footprint of all three buildings is clearly visible and comparable in terms of power. A strong component around $+30 \mathrm{~Hz}$ can be attributed to the specular reflection taking place on building faces. As visible, the architecture of the facades has a strong impact on the scattered power and the second building gives rise to high specular reflections and no backscattering while first and third buildings have lower specular reflections but significant backscattering contributions around $-30 \mathrm{~Hz}$.

\section{Wide-Band Validation Example}

5.1. Qualitative Observation of Channel IR. To complete the narrow-band validation performed in the previous section, 

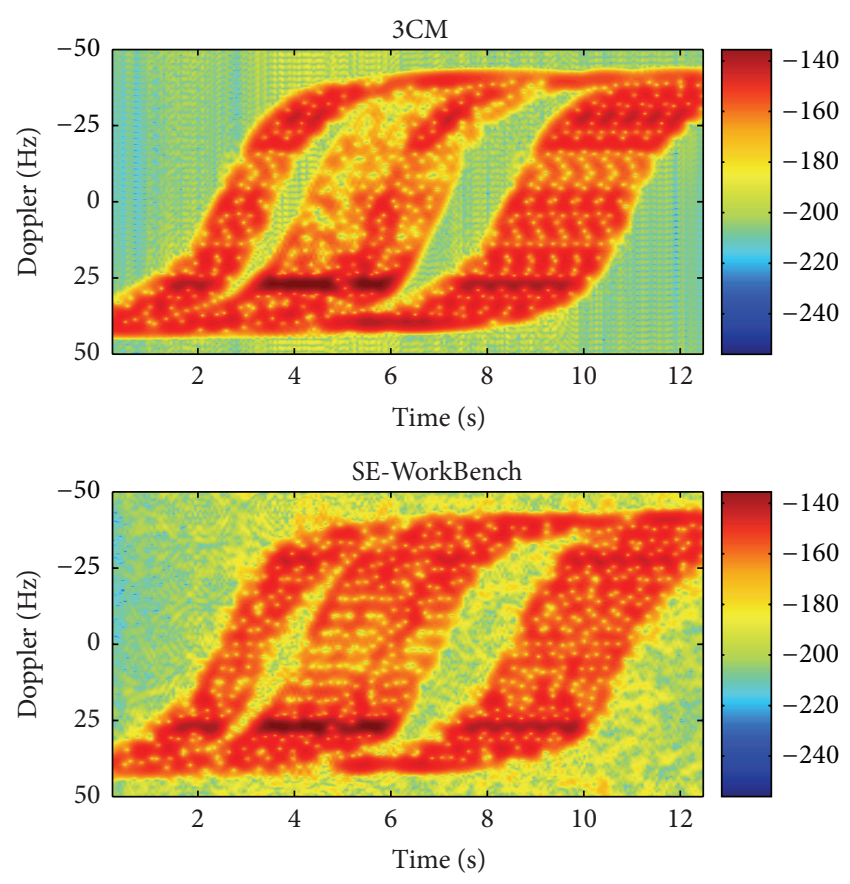

FIGURE 12: Spectrogram along receiver trajectory.

a wide-band validation is here presented. All parameters from the previously described urban scenario are kept constant. The first validation performed in this section deals with a qualitative observation of instantaneous CIRs presented in Figure 13. For this snapshot, the receiver was at position $y=$ $10 \mathrm{~m}$ as presented on top of Figure 11. In Figure 13 the $X$-axis represents the relative delay of each echo with respect to the LOS, the $Y$-axis represents the absolute Doppler of each echo, and colour axis represents the relative power of each echo. The power scale has been truncated to the zone of interest from $0 \mathrm{~dB}$ to $-40 \mathrm{~dB}$ as explained in Section 4.1. The wideband technique used to produce pseudo-CIRs using MoM ELSEM3D has been presented in [17] and is not detailed in this paper.

For those simulations, the LOS signal has been taken into account. It is represented by a red dot with null delay and about $25 \mathrm{~Hz}$ Doppler on each three plots in Figure 13. From a qualitative point of view, all three simulations give a comparable result in terms of delay/Doppler channel shape. The main significant difference is that $3 \mathrm{CM}$ seems to underestimate the specular reflection coming from the second building. Note that the specular region is characterised by its Doppler which is equal to the LOS Doppler. In this region, the $3 \mathrm{CM}$ shows echoes of about $-20 \mathrm{~dB}$ while SE-WorkBench and ELSEM3D have stronger echoes of about $-10 \mathrm{~dB}$. This phenomenon can be explained by the fact that the $3 \mathrm{CM}$ has a higher space resolution in terms of environment description, $0.5 \mathrm{~m}$. This results in numerous weak echoes which are radiating in phase to produce constructive interferences. If those echoes were summed together, it would result in fewer but stronger echoes as is the case for the ELSEM3D and SEWorkBench simulations. One other interesting observation is the backscattering contribution, coming from the first
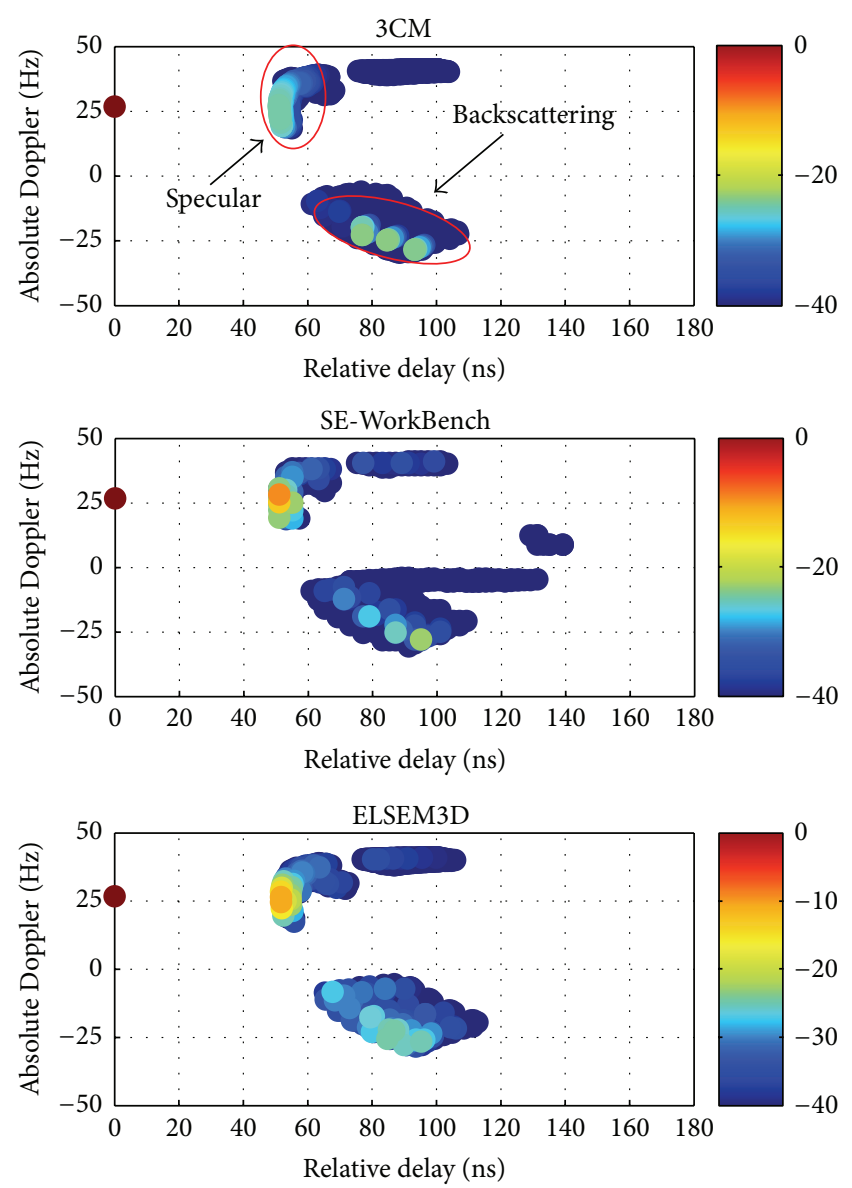

FIGURE 13: Power delay/Doppler channel IR.

building, which can be highlighted in the negative Doppler region. Backscattering contributions seem very punctual for 3CM and SE-WorkBench while they are more diffuse in ELSEM3D simulation.

Regarding the time performances, the presented trajectory has 937 sampling points. This simulation has been performed on a desktop computer with Intel Core2 Duo E8400 CPU $3 \mathrm{GHz}$ and $3 \mathrm{~GB}$ RAM. The following computation times are only indicatives. The $3 \mathrm{CM}$ takes $18.80 \mathrm{~s}$ to simulate all CIRs with more than 2000 rays per CIR. This represents one IR every $20.06 \mathrm{~ms}$. Such computation time is considered as real time for navigation purposes since it is close to typical integration time $(20 \mathrm{~ms})$. On the same computer, the equivalent SE-WorkBench simulation takes about $1730 \mathrm{~s}$, ninety times higher. The ELSEM3D computation time is not representative since the initial computation has been performed on a super computer and CIRs were precalculated.

5.2. Focus on Delay Parameters. From Figure 13, only qualitative observations have been made. To better observe the channel over the whole receiver trajectory two channel parameters relative to the delay have been studied: the MP mean delay and the MP RMS delay spread. Each one is presented in Figure 14 using, respectively, (22) and (23) where 


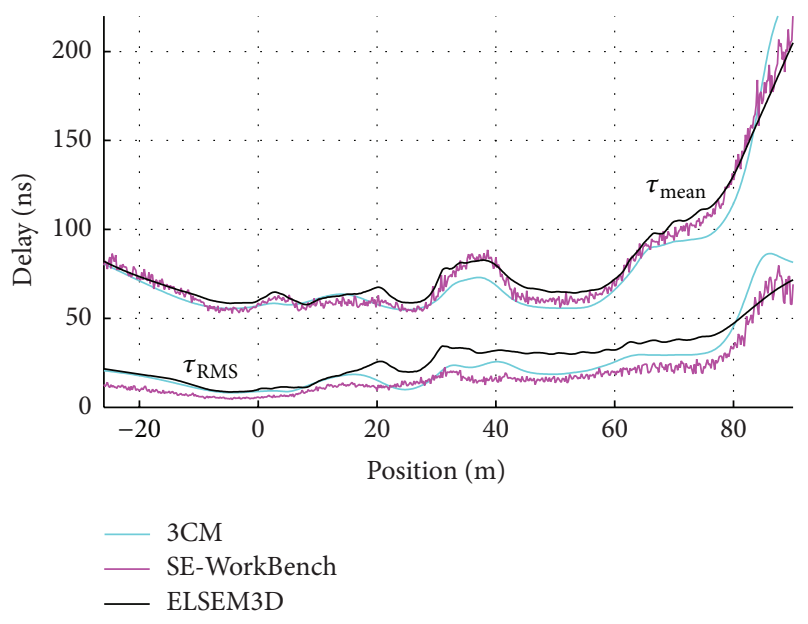

FIGURE 14: $\tau_{\text {mean }}$ and $\tau_{\text {RMS }}$ function of mobile position.

$\Gamma$ represents the power of the echoes and $\tau$ their delay relative to LOS:

$$
\begin{aligned}
\tau_{\text {mean }} & =\frac{\int_{0}^{\infty} \tau \Gamma(\tau) d \tau}{\int_{0}^{\infty} \Gamma(\tau) d \tau}, \\
\tau_{\mathrm{RMS}} & =\sqrt{\frac{\int_{0}^{\infty}\left(\tau-\tau_{\text {mean }}\right)^{2} \Gamma(\tau) d \tau}{\int_{0}^{\infty} \Gamma(\tau) d \tau}} .
\end{aligned}
$$

Since LOS is highly predominant in our simulations, it has been removed to better observe the temporal distribution of the delayed echoes. As visible in Figure 14, the RMS delay spread is low, in the order of few tens of nanoseconds in our simple case. All three delay spread plotted in Figure 14 present the same behaviour. Regarding the mean delay, it can be noticed that it is almost constant from $-5 \mathrm{~m}$ to $60 \mathrm{~m}$. This region corresponds to the specular region of the urban canyon. Observing Figure 13 we can see that 55 ns corresponds more or less to the delay of the specular component. We can conclude from Figure 14 that the main power contribution comes from the specular reflection. It can also be noticed that the mean delay gets higher at the end of the trajectory. This is due to the geometry of the simulation. Since the mobile is facing the LOS, the delay spread is reduced when the buildings are in front of the mobile. On the opposite case, when the buildings are far behind the receiver, echoes have to pass the mobile to reach the buildings before being reflected back to the mobile. This extra path highly increases the delay. Also noticeable is the fact that the pink curve (SE-WorkBench) is not as smooth as the two others. Extra experiments have shown that this phenomenon is due to the MP summation process realised according to the tappeddelay line processing (delay axis discretisation of $2 \mathrm{~ns}$ ) taking place in SE-WorkBench. ELSEM3D and 3CM are not affected since they do not sum any MP contributions.

\section{Conclusion}

This paper has presented a new simplified model to reproduce building scattering in urban environments. The 3CM, 3component model, has been designed according to practical observations made on numerical simulations and experimental measurements. Three different wave propagation mechanisms have been highlighted in this paper on which the $3 \mathrm{CM}$ relies. The specular component is originated from smooth and wide surfaces such as windows and flat walls. The backscattering component is a double bounce reflection mainly taking place on protruding balconies and receding window corners. The incoherent scattering comes from small objects present on facades such as guardrails or rough walls. This three macropropagation decomposition can be also implemented into conventional ray-tracing tools considering minor adjustments.

The 3CM model here presented is oriented toward MP wide-band representation of the channel with a strong emphasis on computation time reduction. Based on a simplified PO algorithm, the 3CM achieves around a onehundred time reduction factor with respect to conventional ray-tracing tools. Since the $3 \mathrm{CM}$ is based on EM model, the 3CM perfectly handles dielectric materials and circular polarisation. It is also frequency scalable and particularly adapted for $\mathrm{L}, \mathrm{S}$, and $\mathrm{C}$ bands without a priori restrictions for higher frequencies, but not yet validated. Typical outputs of the $3 \mathrm{CM}$ are delay, Doppler, DoA, and polarised complex amplitude of each ray. Finally the 3CM is suitable for MIMO (Multiple Inputs Multiple Outputs) studies with transmit/receive diversity, polarisation diversity, or frequency diversity.

In this paper, several validation examples have been provided. First, the EM implementation of all three components taken separately in canonical conditions has been overviewed. Then the total scattered power in the case of one isolated complex facade is followed by the case of a simple urban canyon. Very good agreement has been found considering both MoM and asymptotic GO/PO tools as references. Those comparisons have been performed in terms of power and Doppler spread. Wide-band simulations have also been presented with a qualitative analysis of complex CIRs and delay spread analysis.

The $3 \mathrm{CM}$ is not restricted to the satellite case even if it is designed in such a way. The propagation mechanisms taking place around complex facades are not application dependent and can be used for terrestrial applications. However, due to the geometry imposed by the satellites links, facades are mostly illuminated whereas they are mostly masked in the case of terrestrial links due to grazing geometries. The $3 \mathrm{CM}$ principles have been integrated into a new enhanced simplified channel model handling LOS contributions with blockage and diffractions for better realism in more complex environments. This enhanced channel simulator, SCHUN (Simplified CHannel for Urban Navigation), has been introduced in [4] and is based on the 3CM to synthesise the MP component of the channel. The SCHUN simulator has also been validated against measurement campaigns targeting LMS communication systems in S/C bands and satellite navigation systems from point of view of both channel and system. 


\section{Conflict of Interests}

The authors declare that there is no conflict of interests regarding the publication of this paper.

\section{Acknowledgments}

This paper has been partly carried out in the framework of the European action COST IC0802 "Propagation Tools and Data for Integrated Telecommunication, Navigation, and Earth Observation systems." The authors would also like to particularly thank Paul Thevenon from CNES and Guillaume Carrie from Onera for their participation.

\section{References}

[1] M. Döttling, A. Jahn, D. Didascalou, and W. Wiesbeck, “Twoand three-dimensional ray tracing applied to the land mobile satellite (LMS) propagation channel," IEEE Antennas and Propagation Magazine, vol. 43, no. 6, pp. 27-37, 2001.

[2] ITU, "ITU-R p.681-7 propagation data required for the design of earthspace land mobile telecommunication systems, Propagation data required for the design of Earth-space land mobile telecommunication systems ITU-R P.681-7," 2009.

[3] C. Oestges, Propagation modelling of low earth-orbit satellite personal communication systems [Ph.D. dissertation], Universite Louvain la Neuve, Louvain la Neuve, Belgium, 2000.

[4] M. Ait-Ighil, Enhanced physical-statistical simulator of the land mobile satellite channel for multipath modelling applied to satellite navigation systems [Ph.D. dissertation], Institut Superieur de l'Aeronautique et de l'Espace (ISAE Toulouse), Onera Toulouse, France, 2013, http://www.theses.fr/en/2013ESAE0001.

[5] G. Gougeon, Y. Lostanlen, and L. Maviel, "Coupling a deterministic propagation model with diffuse scattering and urban furniture for small cells," in Proceedings of the 5th European Conference on Antennas and Propagation (EUCAP '11), pp. 3448-3452, April 2011.

[6] G. Calcev, D. Chizhik, B. Göransson et al., "A wideband spatial channel model for system-wide simulations," IEEE Transactions on Vehicular Technology, vol. 56, no. 2, pp. 389-403, 2007.

[7] A. Lehner, Multipath channel modelling for satellite navigation systems [Ph.D. dissertation], Shaker, Erlangen, Germany, 2007.

[8] V. Degli-Esposti, F. Fuschini, E. M. Vitucci, and G. Falciasecca, "Measurement and modelling of scattering from buildings," IEEE Transactions on Antennas and Propagation, vol. 55, no. 1, pp. 143-153, 2007.

[9] P. Pongsilamanee and H. L. Bertoni, "Specular and nonspecular scattering from building facades," IEEE Transactions on Antennas and Propagation, vol. 52, no. 7, pp. 1879-1889, 2004.

[10] S. Mostarshedi, E. Richalot, J. Wiart, and O. Picon, "An assessment of complex scattered electric field through building facade homogenization," in Proceedings of the 5th European Conference on Antennas and Propagation (EUCAP '11), pp. 3293-3296, April 2011.

[11] E. M. Vitucci, F. Mani, V. Degli-Esposti, and C. Oestges, "Polarimetric properties of diffuse scattering from building walls: experimental parameterization of a ray-tracing model," IEEE Transactions on Antennas and Propagation, vol. 60, no. 6, pp. 2961-2969, 2012.

[12] A. Barka, P. Soudais, and D. Volpert, "Scattering from 3-D cavities with a plug and play numerical scheme combining IE,
PDE, and modal techniques," IEEE Transactions on Antennas and Propagation, vol. 48, no. 5, pp. 704-712, 2000.

[13] P. Soudais, H. Steve, and F. Dubois, "Scattering from several test-objects computed by 3-D hybrid IE/PDE methods," IEEE Transactions on Antennas and Propagation, vol. 47, no. 4, pp. 646-653, 1999.

[14] A. Lehner and A. Steingass, "Differences in multipath propagation between urban and suburban environments," in Proceedings of the 4th ESA Workshop on Satellite Navigation User Equipment Technologies (NAVITEC '08), Noordwijk, The Netherlands, December 2008.

[15] H. J. Mametsa, S. Laybros, A. Bergès, P. F. Combes, P. N’Guyen, and P. Pitot, "FERMAT: a high frequency em scattering code from complex scenes including objects and environment," in Proceedings of the 1st European Conference on Antennas and Propagation (EuCAP '06), pp. 1-4, November 2006.

[16] G. T. Ruck, D. E. Barrick, W. D. Stuart, and C. K. Krichbaum, Radar Cross Section Handbook, vol. 1-2, Plenum Press, New York, NY, USA, 1st edition, 1970.

[17] M. Ait-Ighil, J. Lemorton, F. Pérez-Fontán et al., "Simplifying the propagation environment representation for LMS channel modelling," EURASIP Journal on Wireless Communications and Networking, vol. 2012, article 110, 2012.

[18] C. A. Balanis, Advanced Engineering Electromagnetics, John Wiley \& Sons, 1989. 

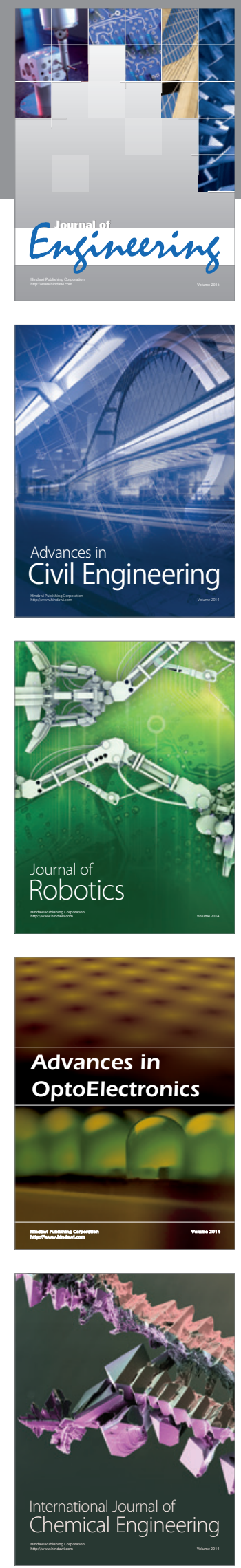

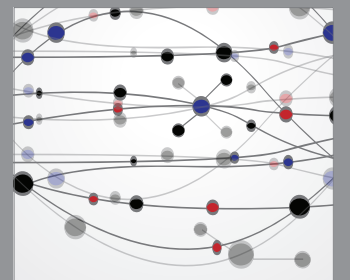

The Scientific World Journal
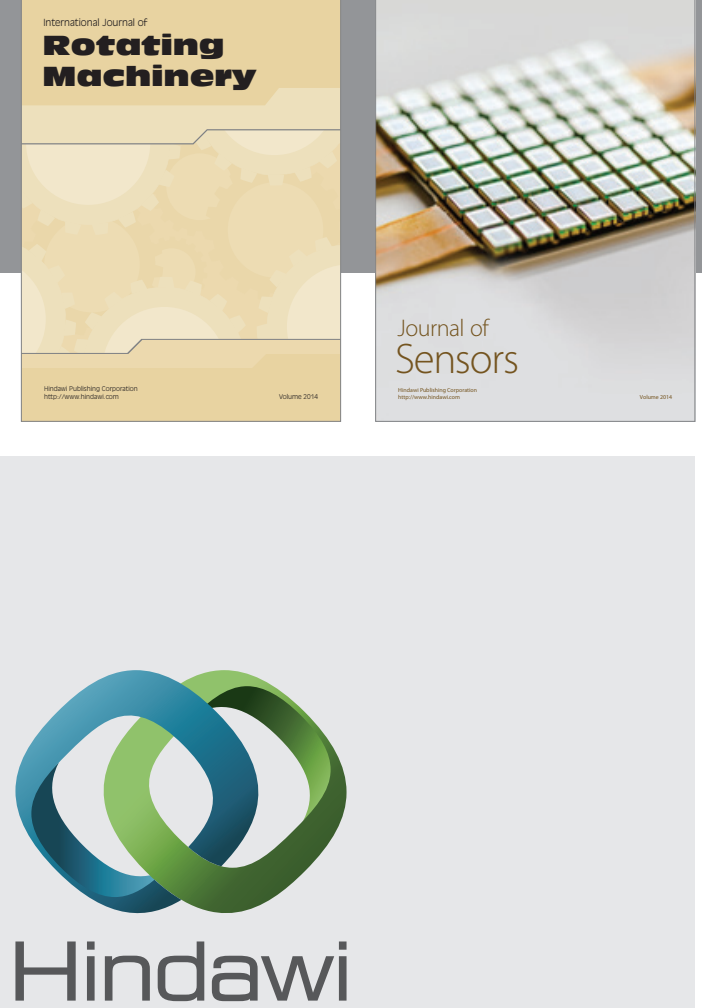

Submit your manuscripts at http://www.hindawi.com
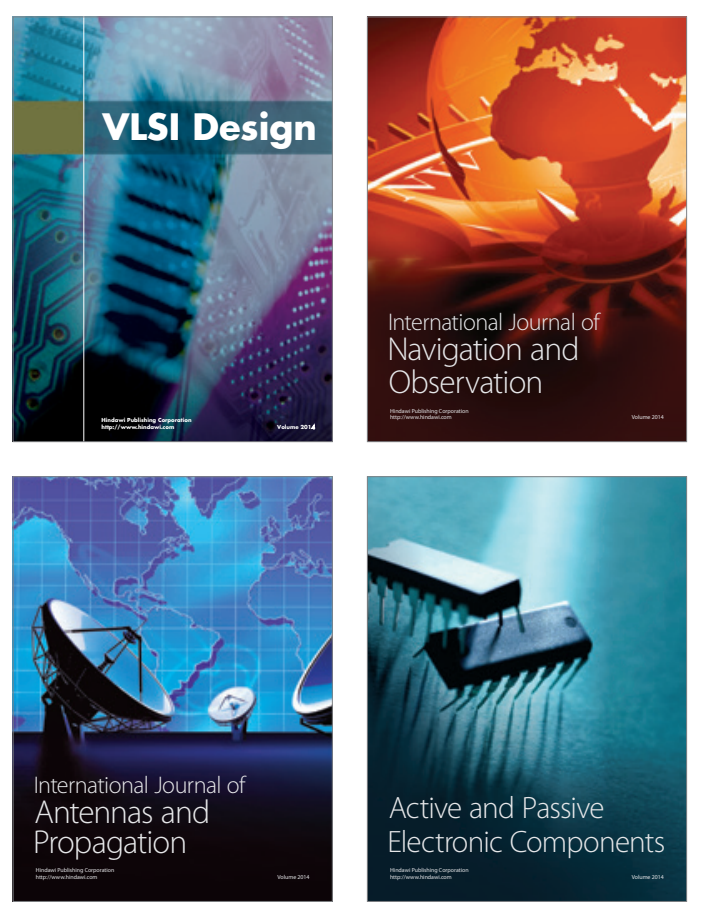
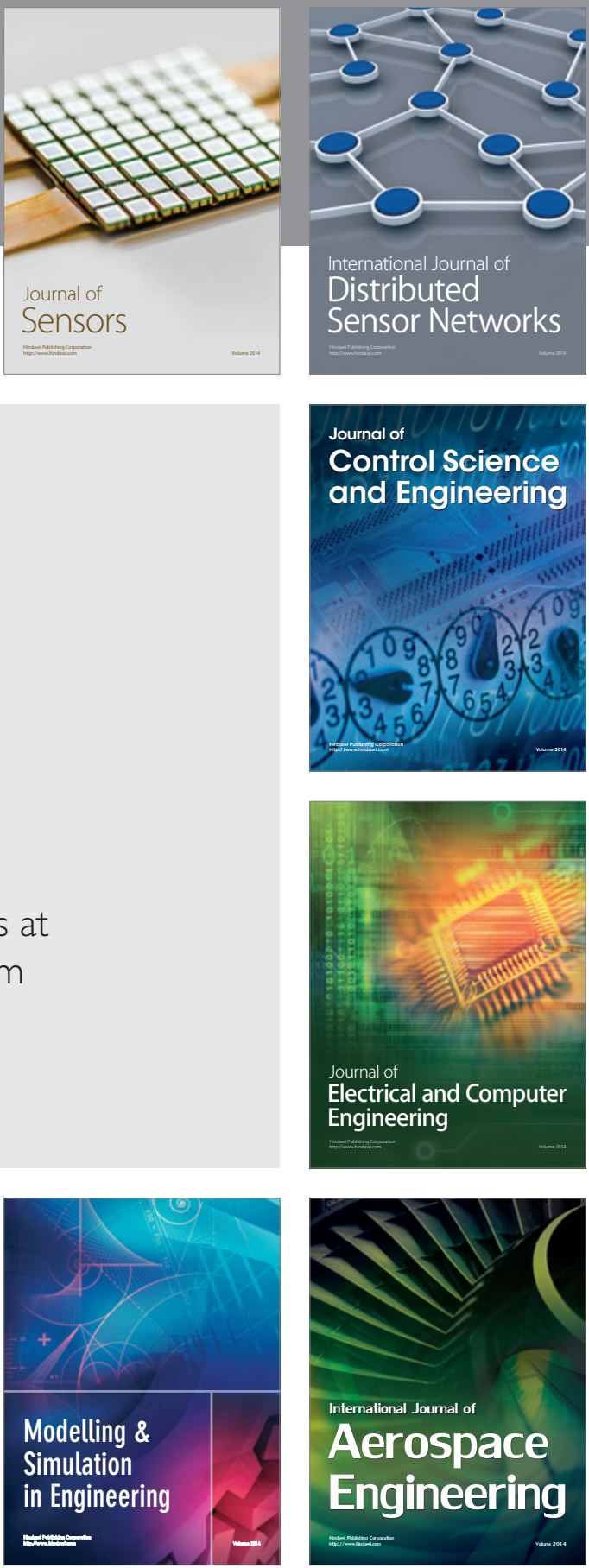

Journal of

Control Science

and Engineering
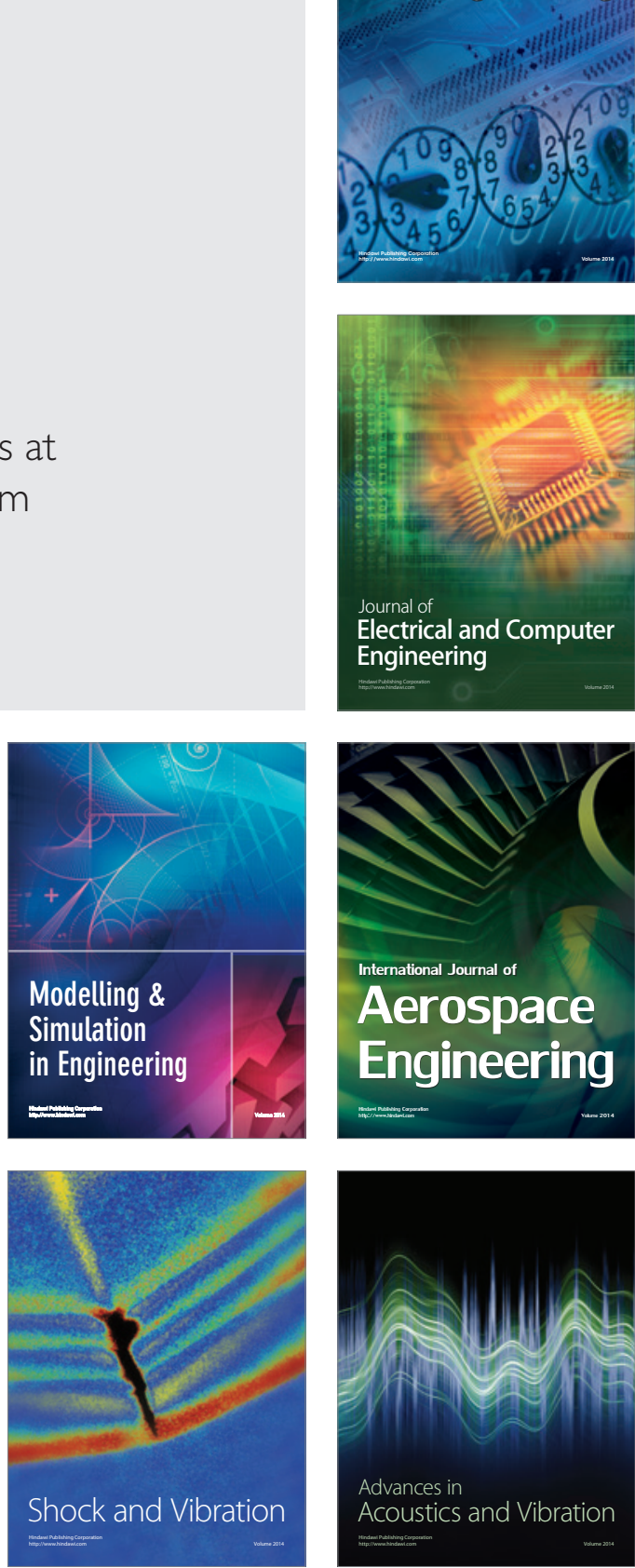